\title{
Effects of framing and missing information in expert and novice judgment
}

\author{
WING HONG LOKE and KAI FOONG TAN \\ National University, Singapore, Singapore
}

\begin{abstract}
We examined the effects of framing and missing information in experts and novices. The present study attempted to show the effect of framing, examined whether experts are less affected by the framing effect than are novices, and examined the differences between experts and novices in the way they respond to complete and missing information. A gambling task was used in which subjects rated their likelihood of taking a gamble on the basis of payoff or chance information, or both. Results showed a significant framing effect; that is, subjects are more likely to take a gamble if it is framed positively than if it is framed negatively. We found that experts and novices are similarly affected by framing but that novices attach more weight to chance information than do experts. Overall, analyses showed no significant difference in the way experts and novices respond to complete and incomplete information.
\end{abstract}

Studies have shown that the way information is presented or framed can affect our judgment (Kahneman \& Tversky, 1979; Tversky \& Kahneman, 1981). Also, judgments and decisions in real situations are usually made with limited information. Individuals utilize different methods of dealing with limited information, such as inferring that high prices imply high quality and vice versa (Huber \& McCann, 1982; Levin, Johnson, \& Faraone, 1984; Yamagishi \& Hill, 1981), imputing values for the missing information by averaging (Slovic \& MacPhillamy, 1974), or assigning less favorable values (Levin, Kim, \& Corry, 1976; Yates, Jagacinski, Carolyn, \& Faber, 1978).

Experts' judgments and decisions can be influenced by the framing effect. Rybash and Roodin (1989) discovered that physicians made different decisions, such as whether to opt for a risky treatment, depending on whether the treatment is expressed in terms of "number of lives saved" (positively framed) or "number of lives lost" (negatively framed). Furthermore, differences may exist between experts and novices under conditions of uncertainty. Uncertainty is defined as a situation in which we are not certain (a probability of less than one) of the outcomes. Von Holstein (1972) compared the forecasts of investment bankers, stock market experts, business administration teachers, and students. The task was to make probability estimates of future prices in the stock market. The bankers' record was the weakest, although no group showed any significant accurate prediction. Studies on the performance of mutual funds showed that their professionally managed portfolios do not consistently outperform the market aver-

This research was supported by National University of Singapore Research Grant RP9000073 to the senior author. Correspondence may be addressed to W. H. Loke, Department of Social Work and Psychology, 10 Kent Ridge Crescent, National University of Singapore, Singapore 0511 . age (e.g., Fabozzi \& Francis, 1979; Fama, 1965), suggesting that experts are susceptible to judgmental biases.

Hence, the present study will also investigate the effects of missing information on judgments-whether experts and novices use similar or different strategies in dealing with complete as well as incomplete (missing) information.

The present paper examines the effects of framing and incomplete information in experts and novices under conditions of uncertainty. Experts will be defined by their academic credentials as well as their job titles (Oskamp, 1965); that is, individuals who have doctoral or master's degrees in the area of finance or business administration and are also finance lecturers. In contrast, novice subjects have neither specialized knowledge in the area nor experience. The three hypotheses examined are: (1) that the framing effect will be replicated using a hypothetical task; (2) that framing effects will be shown in both experts and novices, but experts will be less affected by framing than will novices; and (3) that experts and novices use different strategies in dealing with complete and incomplete information. A gambling task that consists of two dimensions, probability of winning/losing and payoff, is used. The two reasons for using a gambling task are that it is a model for a variety of risktaking economic decisions (i.e., judgments under conditions of uncertainty) and that past research has shown the gambling task to be a valid predictor of behavior outside a laboratory setting (Lichtenstein \& Slovic, 1973).

\section{METHOD}

\footnotetext{
Subjects

Thirteen lecturers, 10 from the Faculty of Business Administration, Singapore Polytechnic, and 3 from the School of Management, National University of Singapore, participated. Twelve third-year business administration undergraduates majoring in finance were also in the pool of experts. The novices were 104 first-year psychology undergraduates (mean age of 20 years; $S D=1.43$ years) from the National University of Singapore. About $75 \%$ are English educated (i.e., having English
} 
as their first language at secondary school level), and the remaining $25 \%$ are Chinese/Malay educated (i.e., having Chinese/Malay as their first language). The difference in sample sizes between experts and novices is due to practical difficulties in obtaining subjects.

\section{Materials}

Two task booklets, one positively framed and the other negatively framed, were used. Each booklet contained 25 gambles and consisted of all combinations of payoff $(\$ 100, \$ 150, \$ 200, \$ 250$, and “blank") and chance of losing/winning $(.05 / .95, .10 / .90, .15 / .85, .20 / .80$, and "'blank"/"blank"). The first page of each booklet contained requests for subjects' biographical data such as age, gender, and educational background. The second page contained general instructions for the task; it was followed by 25 pages of gambles (one per page). The last page of the booklet consisted of a postexperimental questionnaire.

\section{Experimental Design}

The subjects were asked to evaluate a series of 50 gambles. They rated (1) their likelihood to take a gamble (on an interval scale ranging from -3 , very likely to take gamble, to 0 , equally likely to refuse or take gamble, to 3, very likely to refuse gamble); and (2) their confidence in that judgment (measured on a 7-point scale ranging from 1, extremely unconfident, to 7 , extremely confident).

The gambles were depicted by both probability and payoff information or by only one of these sources of information. Probability information was framed in either probability of winning (positive frame) or probability of losing (negative frame). Probabilities of winning are .05 , $.10, .15, .20$, and "blank" in the positive frame, and probabilities of losing are $.80, .85, .90$, and "blank" in the negative frame. Payoff amounts are $\$ 100, \$ 150, \$ 200, \$ 250$, and "blank." "Blank" means no information is given on the particular dimension of chance or payoff information.

Significant $(p<.05)$ and marginal $(.05<p<.10)$ effects are reported. Significant effects are further analyzed using the Newman-Keuls multiple-range tests for comparison of two means.

\section{Instructions}

The subjects were given a page of instructions describing the nature of the hypothetical gambles. They were told that the gambles sometimes represent decisions that have to be made in their everyday lives. They were told that they have $\$ 15$ at the beginning of every gamble, and they can choose to invest it or keep it. Information about the payoff of the gamble as well as the chances of winning or losing are present in the gambling tasks. On some trials, there will be missing information on one or both of these dimensions. The subjects were instructed to go through the gambles as accurately and as quickly as possible. They were also told not to refer back to previous gambles once they are completed. The subjects could ask for clarification before they began the task.

\section{Procedure}

The subjects were presented two task booklets, one positively framed and one negatively framed, with a time interval of a week in between. The combination of the 25 gambles in each booklet was randomized. The order of presentation (positive or negative frame) was counterbalanced.

The subjects were asked to evaluate the likelihood of their taking the gambles on a scale ranging from "very likely to take gamble" to "very likely to refuse gamble." Then the subjects were asked to indicate how confident they were in each of their likelihood ratings. We used a 7-point scale instead of the 20-point scale used by Levin, Chapman, and Johnson (1988) and Loke (1989) because past subjects did not utilize the full range of the 20-point scale (Loke, 1989). Furthermore, instructions were given to encourage the full use of the range of the scale.

\section{Statistics}

Unequal cell analyses of variance (ANOVAs) were used to examine two dependent measures, ratings of likelihood of taking a gamble and ratings of confidence in each likelihood judgment. The main independent variables examined were expertise (experts and novices), framing (positive and negative), payoff (\$100, \$150, \$200, \$250, and “blank"), and chance $(.05 / .95, .10 / .90, .15 / .85, .20 / .80$, and "blank"/“blank"). Also, a secondary independent variable, subjects' experience of frame order (positive-negative and negative-positive conditions) was exam- ined. Order and expertise are between-subject variables, and framing, payoff, and chance are within-subject variables.

A posttest questionnaire was also given to check whether the subjects understood the instructions and the nature of the task. The questionnaire also asked the subjects about the strategies they employed, if any, to reach a decision of how likely they were to take the gambles with either complete or missing information.

\section{RESULTS AND DISCUSSION}

\section{Framing Effects}

Framing effects were statistically significant $[F(1,120)$ $=16.48, p<.001]$. Gambles in the positive-frame condition are lower in likelihood scores $(M=0.76)$ than are those in the negative-frame condition $(M=1.32)$, suggesting that subjects in the positive condition are more likely to take a gamble than are those in the negative condition (see Figure 1). No significant differences in likelihood ratings were shown between experts and novices. Furthermore, the nonsignificance of framing $\times$ expertise $[F(1,120)=0.48]$ suggests that both experts and novices are affected by framing effects. This result is consistent with the framing effects found in judgments of physicians (Rybash \& Roodin, 1989).

In the present study, framing effects were examined in a within-subject design and were found to be significant. This result contrasts with the results of Levin, Johnson, Russo, and Deldin (1985), who reported a significant framing effect for a between-subject design but a nonsignificant framing effect in a within-subject analysis. This nonsignificance may be due to the strength and persistence of the initial frame. Loke (1989) investigated the persistence of initial frame and found that an interval of $48 \mathrm{~h}$ diluted the framing effect for the likelihood response but not for the confidence ratings. In this study, the time interval between the presentation of the two frame conditions is 1 week; similar dilution effects were found for the mean likelihood scores but not for the confidence ratings.

Results showed an interaction of expertise and payoff in the positive-frame condition $[F(4,508)=7.27, p<$ $.001]$. The mean likelihood scores are significantly higher for the novices $(M=5.40)$ than for the experts $(M=$ 1.59). Novices are more likely to refuse a gamble, given payoff information, than are experts, who are more likely to take a gamble. However, this trend does not occur in the negative-frame condition; the interaction between expertise and payoff was not significant $[F(4,488)=0.58]$. However, in the negative frame, results showed a significant interaction between expertise and chance $[F(4,488)$ $=6.43, p<.001]$; that is, given chance information, novices are more likely to refuse a gamble $(M=7.78)$ than are experts $(M=5.14)$. [The interaction for the positive frame was not significant, $F(4,508)=1.02]$.

In both frames, novices are more likely to refuse a gamble than are experts. Specifically, novices are more likely to refuse a gamble when chance information is given in the negative frame. In the positive frame, they refuse a gamble when payoff information is given. Perhaps novices place more weight on the chance information than on the payoff information in determining their likelihood to take a gam- 

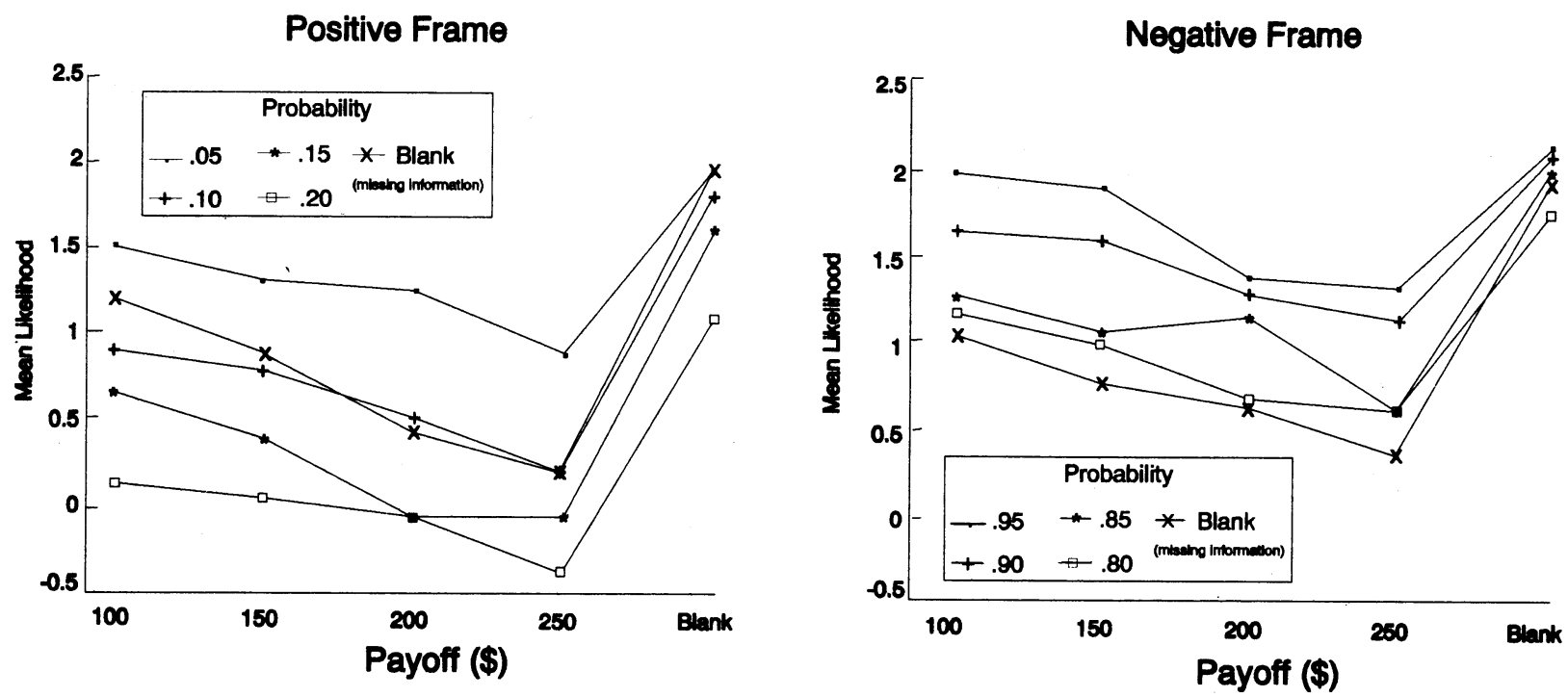

Figure 1. Mean rated likelihood of taking gambles for one- and two-attribute stimuli (i.e., payof amount and probability information). Stimulus variables are payoff amount and probability of winning in the positive frame (left panel) and payoff amount and probability of losing in the negative frame (right panel).

ble. That is, novices attach more weight to one particular dimension (chance information) of a gamble that they consider crucial. In contrast, experts may be able to integrate information better than novices by placing equal or balanced weight on the two dimensions of the gamble.

\section{Missing Information}

The effects of missing information can be observed in Figure 1. In general, when information is missing, it will lead to more extreme likelihood scores. Subjects tend to refuse gambles when there is missing information. The crossed $(X-X)$ line, which represents mean likelihood scores with probability information missing, shows different trends in the two frame conditions. In the left panel of Figure 1, the dotted line lies between probabilities of winning of .05 and .10 , corresponding to a .08 probability of winning. In the right panel of Figure 1 , the crossed line is the lowest line, corresponding to a probability of losing of .85. These extrapolations hold, assuming that the relationships investigated are linear.

In the positive frame, when probability of winning is missing, the mean likelihood ratings tend to increase, suggesting that subjects are more likely to refuse a gamble. In contrast, subjects in the negative frame tend to be more likely to take a gamble when probability of losing is missing. This could mean that the chance information is the source of information for subjects in their likelihood decisions, as supported by Levin et al. (1985). Levin, Johnson, Deldin, Cressey, and Davis (1986) found that in a positive frame, given the source of information missing, subjects would consider this a "loss" and hence would be more likely to refuse a gamble than they would in the negative frame. There is, however, no difference in the way experts and novices respond to missing information.
The order of presentation (positive-negative or negativepositive) was nonsignificant $[F(1,120)=1.04]$, so this "background framing effect" observed by Loke (1989) was not shown here. However, the order of presentation (positive-negative or negative-positive) interacted with framing $[F(1,120)=4.01, p<.05]$. The subjects in the negative-positive condition were more likely to refuse positively framed gambles than were the subjects in the positive-negative condition. This could be due to the persistence of the initial frame. Alternatively, as explained by the prospect theory (Kahneman \& Tversky, 1979), individuals are also assumed to be more responsive to avoiding losses than to achieving gains; hence, a negative frame would allow the same objective information (chance of winning) to be evaluated less favorably than would a positive frame, and the negative frame would tend to persist over time.

The confidence scores across all the factors were nonsignificant except for the interaction of order and expertise $[F(1,120)=5.90, p<.01]$. This only significant interaction could be due to the insensitivity and the "abstractness" of the 7-point confidence scale. The subjects may not have understood the nature of the scale and therefore could not respond accordingly, a view noted in the reports of the posttest questionnaires.

\section{CONCLUSIONS}

In the present study, framing effects were demonstrated in decisions of finance experts and novices and perhaps also in such other areas as advertising, legal decisions, and negotiations. Experts need to be aware that framing bias leads to inaccurate decisions. Alternatively, experts may use the knowledge about framing effects to alter judg- 
ment. For instance, to induce consumers to buy their products, advertisers may choose to highlight favorable information (positive frame) and ignore defects of a product (negative frame). Also, in labor negotiations, Neale and Bazerman (1985) found that inducing a positive frame leads to a decision that is aimed at seeking gains while inducing a negative frame will lead to a decision that is aimed at avoiding losses. Future directions for research should focus on developing strategies that would help experts in overcoming biases.

The hypothetical gambling task may not have been "genuine" enough to elicit true responses from the experts and novices. The experts are individuals who earn high salaries relative to those of the novices, so they may consider the payoffs in the hypothetical gambles unrealistic. Hence, the amount of personal involvement of the experts might have been low, and this could account for the nonsignificant results. If so, this interpretation would contradict Lichtenstein and Slovic (1973), who showed that the gambling task is a valid predictor of behavior outside the laboratory setting, or perhaps the validity is contingent on the type of experts (e.g., gamblers vs. finance professors) performing the simulated gambling task.

The present gambling task, which consists of two attribute stimuli or less, may not be sufficient to test expertise. Phelps and Shanteau (1978) examined "hypothetical" quality assessments of breeding by expert livestock judges in terms of 11 dimensions (e.g., body weight and ham thickness) crucial for breeding. Results showed that the judges were found to make decisions that were reliably influenced by the 11 dimensions.

Future studies could define experts in more rigorous terms. Shanteau (1988) proposed that there are experts, novices, and naive decisionmakers; that experts have reached the pinnacle of their profession, novices have knowledge and experience but have yet to reach the level of expertise, and naive decisionmakers have little, if any decision-making skill in a given area.

\section{REFERENCES}

FabozZi, F. J., \& Francis, J. C. (1979). Mutual fund systematic risk for bull and bear markets. Journal of Finance, 34, 243-250.

FAMA, E. F. (1965). The behavior of stock market prices. Journal of Business, 38, 34-105.

Huber, J., \& MCCANN, J. (1982). The impact of inferential beliefs on product evaluations. Journal of Marketing Research, 19, 324-333.
Kahneman, D., Trersky, A. (1979). Prospect theory: An analysis of decision under risk. Econometrics, 47, 263-291.

Levin, I. P., Chapman, D. P., \& Johnson, R. D. (1988). Confidence in judgments based on incomplete information: An investigation using both hypothetical and real gambles. Journal of Behavioral Decision Making, 1, 29-41.

Levin, I. P., Johnson, R. D., Deldin, P. J., Cressey, L. J., \& Davis, C. D. (1986). Framing effects in decisions with completely and incompletely described alternatives. Organizational Behavior \& Human Decision Processes, 38, 48-64.

LeVin, I. P., Johnson, R. D., \& FARAone, S. V. (1984). Information integration in price-quality tradeoffs: The effects of missing information. Memory \& Cognition, 12, 96-102.

Levin, I. P., Johnson, R. D., Russo, C. P., \& Deldin, P. J. (1985). Framing effects in judgment tasks with varying amounts of information. Organizational Behavior \& Human Decision Processes, 36, 362-377.

Levin, I. P., KIM, K. J., \& CoRRY, F. A. (1976). Invariance of the weight parameter in information integration. Memory \& Cognition, 4, 43-47.

Lichtenstein, S., \& Slovic, P. (1973). Response-induced reversals of preference in gambling: An extended replication in Las Vegas. Journal of Experimental Psychology, 101, 16-20.

LOKE, W. H. (1989). The effects of framing and incomplete information on judgment. Journal of Economic Psychology, 10, 329-341.

Neale, M. A., \& Bazerman, M. H. (1985). The effect of framing and negotiation overconfidence in bargaining behavior and outcomes. Academy of Management Journal, 28, 34-49.

OsKamp, S. (1965). Overconfidence in case-study judgments. Journal of Consulting Psychology, 29, 261-265.

Phelps, R. H., \& Shanteau, J. (1978). Livestock judges: How much information can an expert use? Organizational Behavior \& Human Performance, 21, 209-219.

Rybash, J. M., \& Roodin, P. A. (1989). The framing heuristic influences judgments about younger and older adults' decision to refuse medical treatment. Applied Cognitive Psychology, 3, 171-180.

Shanteau, J. (1988). Psychological characteristics and strategies of expert decision makers. Acta Psychologia, 68, 203-215.

Slovic, P., \& MacPhillamy, D. J. (1974). Dimensional commensurability and cue utilization in comparative judgment. Organizational Behavior \& Human Performance, 11, 172-194.

TVERSKY, A., \& KAHNEMAN, D. (1981). The framing of decisions and the psycholugy of choice. Science, 211, 453-458.

von Holstein, S. (1972). Probabilistic forecasting: An experiment related to stock market. Organizational Behavior \& Human Performance, 83, 139-158.

YAMAGISHI, T., \& HiLL, C. T. (1981). Adding versus averaging models revisited: A test of a path-analytic integration model. Journal of Personality \& Social Psychology, 41, 13-25.

Yates, J. F., Jagacinski, C. M., Carolyn, M., \& Faber, M. D. (1978). Evaluation of partially described multi-attribute options. Organizational Behavior \& Human Performance, 21, 240-251.

(Manuscript received September 24, 1991.) 